\title{
Spheroidal analysis of the generalized MIC-Kepler system
}

\author{
Levon Mardoyan \\ International Center for Advanced Studies, \\ Yerevan State University, \\ 1, Alex Manoogian st., 375025, Yerevan, Armenia
}

\begin{abstract}
This paper deals with the dynamical system that generalizes the MIC-Kepler system. It is shown that the Schrödinger equation for this generalized MIC-Kepler system can be separated in prolate spheroidal coordinates. The coefficients of the interbasis expansions between three bases (spherical, parabolic and spheroidal) are studied in detail. It is found that the coefficients for this expansion of the parabolic basis in terms of the spherical basis, and vice-versa, can be expresses through the Clebsch-Gordan coefficients for the group $S U(2)$ analytically continued to real values of their arguments. The coefficients for the expansions of the prolate spheroidal basis in terms of the spherical and parabolic bases are proved to satisfy three-term recursion relations.
\end{abstract}

\section{Introduction}

The generalized MIC-Kepler system is described by the equation [1]

$$
\frac{1}{2}(-i \nabla-s \mathbf{A})^{2} \psi+\left[\frac{s^{2}}{2 r^{2}}-\frac{1}{r}+\frac{c_{1}}{r(r+z)}+\frac{c_{2}}{r(r-z)}\right] \psi=E \psi,
$$

where $c_{1}$ and $c_{2}$ nonnegative constants, and

$$
\mathbf{A}=\frac{1}{r(r-z)}(y,-x, 0), \quad \text { and } \quad \operatorname{rot} \mathbf{A}=\frac{\mathbf{r}}{r^{3}}
$$

(We use the system of units for which $\hbar=m=e=c=1$.) The monopole number $s$ satisfies the Dirac's rule of charge quantization $s=0, \pm 1 / 2, \pm 1, \ldots$. Each value of $s$ describes its particular generalized MIC-Kepler system. The Schrödinger equation (1.1) for $c_{i}=0(i=1,2)$ and $s \neq 0$ reduces to the Schrödinger equation of the MIC-Kepler system [2] 3. The MIC-Kepler system could be constructed by the reduction of the four-dimensional isotropic oscillator by the use of the so-called Kustaanheimo-Stiefel transformation both on classical and quantum mechanical levels [4. In the similar way, reducing the two- and eight- dimensional isotropic oscillator, one can obtain the two- [5] and five-dimensional [6] analogs of MIC-Kepler system. An infinitely thin solenoid providing the system by the spin $1 / 2$, plays the role of monopole in two-dimensional case, whereas in the five-dimensional case this role is performed by the $S U(2)$ Yang monopole [7, endowing the system by the isospin. All the above-mentioned systems have Coulomb symmetries and are solved in spherical and parabolic coordinates both in discrete and continuous parts of energy spectra 8, 9. There are generalizations of MIC-Kepler systems on three-dimensional sphere 10 and hyperboloid [1] as well. The MIC-Kepler system has been worked out from different points of view in Refs. [12, 13, 14, 15, 16].

At $s=0$, Eq. (1.1) is reduced to the Schrödinger equation for generalized Kepler-Coulomb system [17]. In the case when $s=0$ and $c_{1}=c_{2} \neq 0$, the equation (1.1) reduces to the Hartmann system that has been used for describing axially symmetric systems like ring-shaped molecules [18.

The system described by the Schrödinger equation (1.1) is one of the Smorodinsky-Winternitz type potentials [19. The Smorodinsky-Winternitz type potentials where revived and investigated in the 1990 by Evans [20.

In Ref. [1] it is shown that the variables in Schrödinger equation (1.1) are separated in spherical and parabolic coordinates. In this article is shown that the variables in Eq. (1.1) can be separated in the prolate 
spheroidal coordinates also. The system of spheroidal coordinates a natural system for investigating many problems in mathematical physics (see Ref. 21] and references cited therein). In quantum mechanics, the spheroidal coordinates play an important role because they are appropriate in describing the behavior of a charged particle in the field of two Coulomb centers. The distance $R$ between the centers is a dimensional parameter characterizing the spheroidal coordinates. these coordinates are changed into spherical and parabolic coordinates as $R \rightarrow 0$ and $R \rightarrow \infty$ respectively, if the positions of one Coulomb center and the charged particle are fixed when taking the limits. In this sense, the spheroidal coordinates are more general then the spherical and parabolic coordinates.

\section{Spherical and Parabolic Bases}

For completeness, we here present the solutions of the Schrödinger equation (1.1) found in 11. Eq. (1.1) in the spherical coordinates becomes

$$
\left\{\Delta_{r \theta}+\frac{1}{4 \cos ^{2} \frac{\theta}{2}}\left(\frac{\partial^{2}}{\partial \varphi^{2}}-4 c_{1}\right)+\frac{1}{4 \sin ^{2} \frac{\theta}{2}}\left[\left(\frac{\partial}{\partial \varphi}+2 i s\right)^{2}-4 c_{2}\right]+2\left(E+\frac{1}{r}\right)\right\} \psi=0,
$$

where

$$
\Delta_{r \theta}=\frac{1}{r^{2}} \frac{\partial}{\partial r}\left(r^{2} \frac{\partial}{\partial r}\right)+\frac{1}{\sin \theta} \frac{\partial}{\partial \theta}\left(\sin \theta \frac{\partial}{\partial \theta}\right)
$$

The solution of Eq. [2.1 has the form

$$
\psi_{n j m}^{(s)}\left(r, \theta, \varphi ; \delta_{1}, \delta_{2}\right)=R_{n j}^{(s)}\left(r ; \delta_{1}, \delta_{2}\right) Z_{j m}^{(s)}\left(\theta, \varphi ; \delta_{1}, \delta_{2}\right) .
$$

The functions $Z_{j m}^{(s)}\left(\theta, \varphi ; \delta_{1}, \delta_{2}\right)$ and $R_{n j}^{(s)}\left(r ; \delta_{1}, \delta_{2}\right)$ are givenly the formulae

$$
\begin{gathered}
Z_{j m}^{(s)}\left(\theta, \varphi ; \delta_{1}, \delta_{2}\right)=N_{j m}\left(\delta_{1}, \delta_{2}\right)\left(\cos \frac{\theta}{2}\right)^{m_{1}}\left(\sin \frac{\theta}{2}\right)^{m_{2}} P_{j-m_{+}}^{\left(m_{2}, m_{1}\right)}(\cos \theta) e^{i(m-s) \varphi} \\
R_{n j}^{(s)}(r)=C_{n j}\left(\delta_{1}, \delta_{2}\right)(2 \varepsilon r)^{j+\frac{\delta_{1}+\delta_{2}}{2}} e^{-\varepsilon r} F\left(-n+j+1 ; 2 j+\delta_{1}+\delta_{2}+2 ; 2 \varepsilon r\right)
\end{gathered}
$$

where $P_{n}^{(\alpha, \beta)}(x)$ are the Jacobi polynomials, $F(a ; c ; x)$ is the confluent hypergeometric function, $N_{j m}\left(\delta_{1}, \delta_{2}\right)$ and $C_{n j}\left(\delta_{1}, \delta_{2}\right)$ are normalization constants

$$
\begin{gathered}
N_{j m}\left(\delta_{1}, \delta_{2}\right)=\sqrt{\frac{\left(2 j+\delta_{1}+\delta_{2}+1\right)\left(j-m_{+}\right) ! \Gamma\left(j+m_{+}+\delta_{1}+\delta_{2}+1\right)}{4 \pi \Gamma\left(j-m_{-}+\delta_{1}+1\right) \Gamma\left(j+m_{-}+\delta_{2}+1\right)}}, \\
C_{n j}\left(\delta_{1}, \delta_{2}\right)=\frac{2 \varepsilon^{2}}{\Gamma\left(2 j+\delta_{1}+\delta_{2}+2\right)} \sqrt{\frac{\Gamma\left(n+j+\delta_{1}+\delta_{2}+1\right)}{(n-j-1) !}} .
\end{gathered}
$$

We assume that

$$
\begin{gathered}
\int_{0}^{\pi} \sin \theta Z_{j^{\prime} m^{\prime}}^{(s)}\left(\theta, \varphi ; \delta_{1}, \delta_{2}\right) Z_{j m}^{(s)}\left(\theta, \varphi ; \delta_{1}, \delta_{2}\right) d \theta d \varphi=\delta_{j j^{\prime}} \delta_{m m^{\prime}} \\
\int_{0}^{\infty} r^{2} R_{n j}^{(s)}\left(r ; \delta_{1}, \delta_{2}\right) R_{n j}^{(s)}\left(r ; \delta_{1}, \delta_{2}\right) d r=\delta_{n n^{\prime}}
\end{gathered}
$$


and denote by the $\varepsilon$ the following expression

$$
\varepsilon=\sqrt{-2 E}=\frac{1}{n+\frac{\delta_{1}+\delta_{2}}{2}} .
$$

The energy spectrum has the form

$$
E \equiv E_{n}^{(s)}=-\frac{1}{2\left(n+\frac{\delta_{1}+\delta_{2}}{2}\right)^{2}}
$$

and the quantum numbers $m$ and $j$ run through the values: $m=-j,-j+1, \ldots, j-1, j$ and

$$
j=\frac{|m+s|+|m-s|}{2}, \frac{|m+s|+|m-s|}{2}+1, \ldots
$$

We make the following notation also $m_{ \pm}=(|m+s| \pm|m-s|) / 2$ and

$$
m_{1}=|m-s|+\delta_{1}=\sqrt{(m-s)^{2}+4 c_{1}}, \quad m_{2}=|m+s|+\delta_{2}=\sqrt{(m+s)^{2}+4 c_{2}}
$$

The wave functions (2.3) are the eigenfunctions of commuting operators $\hat{M}$ and $\hat{J}_{z}$, moreover and

$$
\hat{M} \psi_{n j m}^{(s)}\left(r, \theta, \varphi ; \delta_{1}, \delta_{2}\right)=\left(j+\frac{\delta_{1}+\delta_{2}}{2}\right)\left(j+\frac{\delta_{1}+\delta_{2}}{2}+1\right) \psi_{n j m}^{(s)}\left(r, \theta, \varphi ; \delta_{1}, \delta_{2}\right),
$$

where

$$
\hat{M}=\hat{J}^{2}+\frac{2 c_{1}}{1+\cos \theta}+\frac{2 c_{2}}{1-\cos \theta} .
$$

Here $\hat{J}^{2}$ is the square of the angular momentum [2]

$$
\hat{\mathbf{J}}=\mathbf{r} \times(-i \nabla-s \mathbf{A})-s \frac{\mathbf{r}}{r}
$$

$\hat{J}_{z}=s-i \partial / \partial \varphi$ its $z$-component and $\hat{J}_{z} \psi=m \psi$. The operator $\hat{M}$ is written in the Cartesian coordinates as

$$
\hat{M}=-r^{2} \Delta+x_{i} x_{j} \frac{\partial^{2}}{\partial x_{i} \partial x_{j}}+2 x_{i} \frac{\partial}{\partial x_{i}}+\frac{2 i s r}{r-z}\left(x \frac{\partial}{\partial y}-y \frac{\partial}{\partial x}-i s-i \frac{c_{2}}{s}\right)+\frac{2 c_{1} r}{r+z} .
$$

Let us consider the generalized MIC-Kepler system in the parabolic coordinates coordinates $\xi, \eta \in$ $[0, \infty), \varphi \in[0,2 \pi)$, defined by the formulae

$$
x=\sqrt{\xi \eta} \cos \varphi, \quad y=\sqrt{\xi \eta} \sin \varphi, \quad z=\frac{1}{2}(\xi-\eta),
$$

In this coordinates the differential elements of length and volume read

$$
d l^{2}=\frac{\xi+\eta}{4}\left(\frac{d \xi^{2}}{\xi}+\frac{d \eta^{2}}{\eta}\right)+\xi \eta d \varphi^{2}, \quad d V=\frac{1}{4}(\xi+\eta) d \xi d \eta d \varphi
$$

while the Laplace operator looks like

$$
\Delta=\frac{4}{\xi+\eta}\left[\frac{\partial}{\partial \xi}\left(\xi \frac{\partial}{\partial \xi}\right)+\frac{\partial}{\partial \eta}\left(\eta \frac{\partial}{\partial \eta}\right)\right]+\frac{1}{\xi \eta} \frac{\partial^{2}}{\partial \varphi^{2}} .
$$

The substitution

$$
\psi(\xi, \eta, \varphi)=\Phi_{1}(\xi) \Phi_{2}(\eta) \frac{e^{i(m-s) \varphi}}{\sqrt{2 \pi}}
$$


separates the variables in the Schrödinger equation and we arrive at the following system of equations

$$
\begin{aligned}
& \frac{d}{d \xi}\left(\xi \frac{d \Phi_{1}}{d \xi}\right)+\left[\frac{E}{2} \xi-\frac{m_{1}^{2}}{4 \xi}+\frac{1}{2} \beta+\frac{1}{2}\right] \Phi_{1}=0, \\
& \frac{d}{d \eta}\left(\eta \frac{d \Phi_{2}}{d \eta}\right)+\left[\frac{E}{2} \eta-\frac{m_{2}^{2}}{4 \eta}-\frac{1}{2} \beta+\frac{1}{2}\right] \Phi_{2}=0,
\end{aligned}
$$

where $\beta$ - is the separation constant.

These equations are analogous with the equations of the hydrogen atom in the parabolic coordinates 22 . Thus, we get

$$
\psi_{n_{1} n_{2} m}^{(s)}\left(\xi, \eta, \varphi ; \delta_{1}, \delta_{2}\right)=\sqrt{2} \varepsilon^{2} \Phi_{n_{1} m_{1}}(\xi) \Phi_{n_{2} m_{2}}(\eta) \frac{e^{i(m-s) \varphi}}{\sqrt{2 \pi}}
$$

where

$$
\Phi_{n_{i} m_{i}}(x)=\frac{1}{\Gamma\left(m_{i}+1\right)} \sqrt{\frac{\Gamma\left(n_{i}+m_{i}+1\right)}{\left(n_{i}\right) !}} e^{-\frac{\varepsilon x}{2}}(\varepsilon x)^{\frac{m_{i}}{2}} F\left(-n_{i} ; m_{i}+1 ; \varepsilon x\right) .
$$

Here $n_{1}$ and $n_{2}$ are nonnegative integers

$$
n_{1}=-\frac{|m-s|+\delta_{1}+1}{2}+\frac{\beta+1}{2 \varepsilon}, \quad n_{2}=-\frac{|m+s|+\delta_{2}+1}{2}-\frac{\beta-1}{2 \varepsilon} .
$$

From the last relations, taking into account (2.11), we get that the parabolic quantum numbers $n_{1}$ and $n_{2}$ are connected with the principal quantum number $n$ as follows

$$
n=n_{1}+n_{2}+\frac{|m-s|+|m+s|}{2}+1
$$

Excluding the energy $E$ from Eqs. (5.4) and (5.5), we obtain the additional integral of motion

$$
\begin{aligned}
\hat{X}= & \frac{2}{\xi+\eta}\left[\xi \frac{\partial}{\partial \eta}\left(\eta \frac{\partial}{\partial \eta}\right)-\eta \frac{\partial}{\partial \xi}\left(\xi \frac{\partial}{\partial \xi}\right)\right]+\frac{\xi-\eta}{2 \xi \eta} \frac{\partial^{2}}{\partial \varphi^{2}}-i s \frac{\xi^{2}+\eta^{2}}{\xi \eta(\xi+\eta)} \frac{\partial}{\partial \varphi}- \\
& -s^{2} \frac{\xi-\eta}{2 \xi \eta}+\frac{2 c_{1} \eta}{\xi(\xi+\eta)}-\frac{2 c_{2} \xi}{\eta(\xi+\eta)}+\frac{\xi-\eta}{\xi+\eta}
\end{aligned}
$$

with the eigenvalues

$$
\beta=\varepsilon\left(n_{1}-n_{2}+\frac{|m-s|-|m+s|+\delta_{1}-\delta_{2}}{2}\right)
$$

and eigenfunctions $\psi_{n_{1} n_{2} m}^{(s)}\left(\xi, \eta, \varphi ; \delta_{1}, \delta_{2}\right)$, i.e.

$$
\hat{X} \psi_{n_{1} n_{2} m}^{(s)}\left(\xi, \eta, \varphi ; \delta_{1}, \delta_{2}\right)=\beta \psi_{n_{1} n_{2} m}^{(s)}\left(\xi, \eta, \varphi ; \delta_{1}, \delta_{2}\right) .
$$

In Cartesian coordinates, the operator $\hat{X}$ can be rewritten as

$$
\begin{aligned}
\hat{X}= & z\left(\frac{\partial^{2}}{\partial x^{2}}+\frac{\partial^{2}}{\partial y^{2}}\right)-x \frac{\partial^{2}}{\partial x \partial z}-y \frac{\partial^{2}}{\partial y \partial z}-i s \frac{r+z}{r(r-z)}\left(x \frac{\partial}{\partial y}-y \frac{\partial}{\partial x}\right)- \\
& -\frac{\partial}{\partial z}-s^{2} \frac{r+z}{r(r-z)}+c_{1} \frac{r-z}{r(r+z)}-c_{2} \frac{r+z}{r(r-z)}+\frac{z}{r},
\end{aligned}
$$

so that it immediately follows that $\hat{X}$ is connected to the $z$-component $\hat{I}_{z}$ of the analog of the Runge-Lenz vector

via

$$
\hat{\mathbf{I}}=\frac{1}{2}[(-i \nabla-s \mathbf{A}) \times \hat{\mathbf{J}}-\hat{\mathbf{J}} \times(-i \nabla-s \mathbf{A})]+\frac{\mathbf{r}}{r}
$$

$$
\hat{X}=\hat{I}_{z}+c_{1} \frac{r-z}{r(r+z)}-c_{2} \frac{r+z}{r(r-z)}
$$

and coincides with $\hat{I}_{z}$ when $c_{1}=c_{2}=0$. 


\section{Bi-Orthogonality of the Radial Wavefunctions}

We shall prove that along with the condition (2.9) the radial wave functions $R_{n j}^{(s)}\left(r ; \delta_{1}, \delta_{2}\right)$ satisfy the following additional orthogonality condition

$$
I_{j j^{\prime}}=\int_{0}^{\infty} R_{n j^{\prime}}^{(s)}\left(r ; \delta_{1}, \delta_{2}\right) R_{n j}^{(s)}\left(r ; \delta_{1}, \delta_{2}\right) d r=\frac{2}{\left(n+\frac{\delta_{1}+\delta_{2}}{2}\right)^{3}} \frac{\delta_{j j^{\prime}}}{2 j+\delta_{1}+\delta_{2}+1} .
$$

This new relation is used in the next Section to derive interbasis expansions. It can be proved as follows.

In the integral appearing in (3.1), we substitute explicit expressions (2.5) for $R_{n j}^{(s)}\left(r ; \delta_{1}, \delta_{2}\right)$ and $R_{n j^{\prime}}^{(s)}\left(r ; \delta_{1}, \delta_{2}\right)$. Then, we take the confluent hypergeometric function in (2.5) as an finite sum

$$
F\left(-n+j+1 ; 2 j+\delta_{1}+\delta_{2}+2 ; 2 \varepsilon r\right)=\sum_{p=0}^{n-j-1} \frac{(-n+j+1) p}{p !\left(2 j+\delta_{1}+\delta_{2}+1\right)_{p}}(2 \varepsilon r)^{p}
$$

and perform the integration term by term with the help of the formula 22$]$

$$
\int_{0}^{\infty} e^{-\lambda x} x^{\nu} F(\alpha, \gamma ; k x) d x=\frac{\Gamma(\nu+1)}{\lambda^{\nu+1}}{ }_{2} F_{1}\left(\alpha, \nu+1, \gamma ; \frac{k}{\lambda}\right) \text {. }
$$

Applying the formula

$$
{ }_{2} F_{1}(a, b ; c ; 1)=\frac{\Gamma(c) \Gamma(c-a-b)}{\Gamma(c-a) \Gamma(c-b)}
$$

for the hypergeometric function, we obtain

$$
\begin{aligned}
I_{j j^{\prime}}= & \frac{\Gamma\left(j+j^{\prime}+\delta_{1}+\delta_{2}+1\right)}{\Gamma\left(2 j+\delta_{1}+\delta_{2}+2\right)}\left[\frac{\left.\Gamma\left(n+j+\delta_{1}+\delta_{2}+1\right)\right)}{(n-j-1) !\left(n-j^{\prime}-1\right) ! \Gamma\left(n+j^{\prime}+\delta_{1}+\delta_{2}+1\right)}\right]^{1 / 2} \times \\
& \times \frac{2}{\left(n+\frac{\delta_{1}+\delta_{2}}{2}\right)^{3}} \sum_{p=0}^{n-j-1} \frac{(-n+j+1)_{p}\left(j+j^{\prime}+\delta_{1}+\delta_{2}+1\right)_{p}}{p !\left(2 j+\delta_{1}+\delta_{2}+2\right)_{p}} \frac{\Gamma(n-j-p)}{\Gamma\left(j^{\prime}-j-p+1\right)} .
\end{aligned}
$$

By introducing the formula [23]

$$
\frac{\Gamma(z)}{\Gamma(z-n)}=(-1)^{n} \frac{\Gamma(-z+n+1)}{\Gamma(-z+1)}
$$

into (3.6), the sum over $p$ can be expressed in terms of the ${ }_{2} F_{1}$ Gauss hypergeometric function of argument 1. We thus obtain

$$
\begin{aligned}
I_{j j^{\prime}}= & \frac{1}{j+j^{\prime}+\delta_{1}+\delta_{2}+1}\left[\frac{\left.(n-j-1) ! \Gamma\left(n+j+\delta_{1}+\delta_{2}+1\right)\right)}{\left(n-j^{\prime}-1\right) ! \Gamma\left(n+j^{\prime}+\delta_{1}+\delta_{2}+1\right)}\right]^{1 / 2} \times \\
& \times \frac{2}{\left(n+\frac{\delta_{1}+\delta_{2}}{2}\right)^{3}} \frac{1}{\Gamma\left(j-j^{\prime}+1\right) \Gamma\left(j^{\prime}-j+1\right)} .
\end{aligned}
$$

Equation (3.1) then easily follows from since $\left[\Gamma\left(j-j^{\prime}+1\right) \Gamma\left(j^{\prime}-j+1\right)\right]^{-1}=\delta_{j j^{\prime}}$.

The result provided by Eq. (3.1) generalizes the one for the hydrogen atom [24]. Indeed, orthogonality properties similar to (3.1) hold for the Kepler-Coulomb system and harmonic oscillator in $f$-dimensional spaces $(f \geq 2)[24$. 


\section{Interbasis Expansion Between Parabolic and Spherical Bases}

The connection between spherical $(r, \theta, \varphi)$ and parabolic $(\xi, \eta, \varphi)$ coordinates is

$$
\xi=r(1+\cos \theta), \quad \eta=r(1+\cos \theta), \quad \varphi(\text { parabolic })=\varphi(\text { spherical }) .
$$

Now, we can write, for fixed value energy $E_{n}^{(s)}$, the parabolic bound states (2.22) as a coherent quantum mixture of the spherical bound states (2.3)

$$
\psi_{n_{1} n_{2} m}^{(s)}\left(\xi, \eta, \varphi ; \delta_{1}, \delta_{2}\right)=\sum_{j=m_{+}}^{n-1} W_{n_{1} n_{2} m s}^{j}\left(\delta_{1}, \delta_{2}\right) \psi_{n j m}^{(s)}\left(r, \theta, \varphi ; \delta_{1}, \delta_{2}\right) .
$$

By virtue of Eq. (4.1), the left-hand side of (4.2) can be rewritten in spherical coordinates. Then, by substituting $\theta=0$ in the so-obtained equation and by taking into account that

$$
P_{n}^{(\alpha, \beta)}(1)=\frac{(\alpha+1)_{n}}{n !}
$$

we get an equation that depends only on the variable $r$. Thus, we can use the orthogonality relation (3.1) on the quantum number $j$. This yields

$$
W_{n_{1} n_{2} m s}^{j}\left(\delta_{1}, \delta_{2}\right)=\frac{\sqrt{\left(2 j+\delta_{1}+\delta_{2}+1\right)\left(j-m_{+}\right) !}}{\Gamma\left(m_{1}+1\right) \Gamma\left(2 j+\delta_{1}+\delta_{2}+2\right)} E_{n_{1} n_{2}}^{j m s} K_{j m s}^{n n_{1}}
$$

where

$$
E_{n_{1} n_{2}}^{j m s}=\left[\frac{\Gamma\left(j-m_{-}+\delta_{1}+1\right) \Gamma\left(n_{1}+m_{1}+1\right) \Gamma\left(n_{2}+m_{2}+1\right) \Gamma\left(n+j+\delta_{1}+\delta_{2}+1\right)}{\left(n_{1}\right) !\left(n_{2}\right) !(n-j-1) ! \Gamma\left(j+m_{-}+\delta_{2}+1\right) \Gamma\left(j+m_{+}+\delta_{1}+\delta_{2}+1\right)}\right]^{\frac{1}{2}},
$$

and

$$
K_{j m s}^{n n_{1}}=\int_{0}^{\infty} e^{-x} x^{j+m_{1}+\delta_{1}+\delta_{2}} F\left(-n_{1} ; m_{1}+1 ; x\right) F\left(-n+j+1 ; 2 j+\delta_{1}+\delta_{2}+2 ; x\right) d x .
$$

To calculate the integral $K_{j m s}^{n n_{1}}$, it is sufficient to write the confluent hypergeometric function $F\left(-n_{1} ; m_{1}+1 ; x\right)$ as a series, integrate according to (3.3) and use the formula (3.4) for the summation of the hypergeometric function ${ }_{2} F_{1}$. We thus obtain

$$
\begin{aligned}
K_{j m s}^{n n_{1}}=\frac{\left(n-m_{+}-1\right) ! \Gamma\left(2 j+\delta_{1}+\delta_{2}+2\right) \Gamma\left(j+m_{+}+\delta_{1}+\delta_{2}+1\right)}{\left(j-m_{+}\right) ! \Gamma\left(n+j+\delta_{1}+\delta_{2}+1\right)} \times \\
\times{ }_{3} F_{2}\left\{\begin{array}{l}
-n_{1},-j+m_{+}, j+m_{+}+\delta_{1}+\delta_{2}+1 \\
m_{1}+1,-n+m_{+}+1
\end{array} \mid 1\right\} .
\end{aligned}
$$

The introduction of (4.5) and (4.7) into (5.9) gives

$$
\begin{array}{r}
W_{n_{1} n_{2} m s}^{j}\left(\delta_{1}, \delta_{2}\right)=\sqrt{\frac{\left(2 j+\delta_{1}+\delta_{2}+1\right) \Gamma\left(n_{1}+m_{1}+1\right) \Gamma\left(n_{2}+m_{2}+1\right)}{\left(n_{1}\right) !\left(n_{2}\right) !(n-j-1) !\left(j-m_{+}\right) ! \Gamma\left(j+m_{-}+\delta_{2}+1\right)}} \times \\
\times \frac{\left(n-m_{+}-1\right) !}{\Gamma\left(m_{1}+1\right)} \sqrt{\frac{\Gamma\left(j-m_{-}+\delta_{1}+1\right) \Gamma\left(j+m_{+}+\delta_{1}+\delta_{2}+1\right)}{\Gamma\left(n+j+\delta_{1}+\delta_{2}+1\right)}} \times \\
\times{ }_{3} F_{2}\left\{\begin{array}{l}
-n_{1},-j+m_{+}, j+m_{+}+\delta_{1}+\delta_{2}+1 \\
m_{1}+1,-n+m_{+}+1
\end{array} \mid 1\right\} .
\end{array}
$$


The next step is to show that the interbasis coefficients (5.10) are indeed a continuation on the real line of the Clebsch-Gordan coefficients for the group $S U(2)$. It is known that the Clebsch-Gordan coefficient $C_{a, \alpha ; b, \beta}^{c, \gamma}$ can be written as 25 ]

$$
\begin{aligned}
& C_{a \alpha ; b \beta}^{c \gamma}=\left[\frac{(2 c+1)(a+\alpha) !(c+\gamma) !}{(a-\alpha) !(c-\gamma) !(a+b+c+1) !(a+b-c) !(a-b+c) !(b-a+c) !}\right]^{1 / 2} \times \\
& \times(-1)^{a-\alpha} \delta_{\gamma, \alpha+\beta} \frac{(a+b-\gamma) !(b+c-\alpha) !}{\sqrt{(b-\beta) !(b+\beta) !}} 3 F_{2}\left\{\begin{array}{l}
-a-b-c-1,-a+\alpha,-c+\gamma \\
-a-b+\gamma,-b-c+\alpha
\end{array} \mid 1\right\} .
\end{aligned}
$$

By using the formula 26

$$
{ }_{3} F_{2}\left\{\begin{array}{l}
s, s^{\prime},-N \\
t^{\prime}, 1-N-t
\end{array} \mid 1\right\}=\frac{(t+s)_{N}}{(t)_{N}}{ }_{3} F_{2}\left\{\begin{array}{l}
s, t^{\prime}-s^{\prime},-N \\
t^{\prime}, t+s
\end{array} \mid 1\right\}
$$

equation (4.10) can be rewritten in the form

$$
\begin{gathered}
C_{a \alpha ; b \beta}^{c \gamma}=\left[\frac{(2 c+1)(b-a+c) !(a+\alpha) !(b+\beta) !(c+\gamma) !}{(b-\beta) !(c-\gamma) !(a+b-c) !(a-b+c) !(a+b+c+1) !}\right]^{1 / 2} \times \\
\quad \times \delta_{\gamma, \alpha+\beta} \frac{(-1)^{a-\alpha}}{\sqrt{(a-\alpha) !}} \frac{(a+b-\gamma) !}{(b-a+\gamma) !}{ }^{2} F_{2}\left\{\begin{array}{l}
-a+\alpha, c+\gamma+1,-c+\gamma \\
\gamma-a-b, b-a+\gamma+1
\end{array} \mid 1\right\} .
\end{gathered}
$$

Finally, comparing (4.12) and (5.10), we obtain the representation

$$
W_{n_{1} n_{2} m s}^{j}\left(\delta_{1}, \delta_{1}\right)=(-1)^{n_{1}} C_{\frac{n+m_{-}+\delta_{2}-1}{2}}^{j+\frac{\delta_{1}+\delta_{2}}{2}, \frac{m_{2}+n_{2}-n_{1}}{2}} ; \frac{n-m_{-}+\delta_{1}-1}{2}, \frac{m_{1}+n_{1}-n_{2}}{2} .
$$

Equation (4.12) proves that the coefficients for the expansion of the parabolic basis in terms of the spherical basis are nothing but the analytical continuation, for real values of their arguments, of the $S U(2)$ ClebschGordan coefficients.

The inverse of Eq. (4.2), namely

$$
\psi_{n j m}^{(s)}\left(r, \theta, \varphi ; \delta_{1}, \delta_{2}\right)=\sum_{n_{1}=0}^{n-m_{+}-1} \tilde{W}_{n j m s}^{n_{1}}\left(\delta_{1}, \delta_{2}\right) \psi_{n_{1} n_{2} m}^{(s)}\left(\xi, \eta, \varphi ; \delta_{1}, \delta_{2}\right),
$$

is an immediate consequence of the orthonormality property of the $S U(2)$ Clebsch-Gordan coefficients. The expansion coefficients in (4.13) are thus given by

$$
\tilde{W}_{n j m s}^{n_{1}}\left(\delta_{1}, \delta_{2}\right)=(-1)^{n_{1}} C_{\frac{n+m_{-}+\delta_{2}-1}{2}}^{j+\frac{\delta_{1}+\delta_{2}}{2},}, \frac{m_{1}+m_{2}}{2}
$$

and may be expressed in terms of the ${ }_{3} F_{2}$ function through (4.10) or 4.12).

\section{Prolate Spheroidal Basis}

We now pass to the prolate spheroidal coordinates

$$
x=\frac{R}{2} \sqrt{\left(\mu^{2}-1\right)\left(1-\nu^{2}\right)} \cos \varphi, \quad y=\frac{R}{2} \sqrt{\left(\mu^{2}-1\right)\left(1-\nu^{2}\right)} \sin \varphi, \quad z=\frac{R}{2}(\mu \nu+1),
$$

where $\mu \in[0 ; \infty), \nu \in[-1 ; 1], \varphi \in[0,2 \pi)$, and $R \in[0 ; \infty)$. The parameter $R$ is the interfocus distance, and in the limits where $R \rightarrow 0$ and $R \rightarrow \infty$, the prolate spheroidal coordinates give back the spherical coordinates and the parabolic coordinates, respectively [21, 27]. 
The Laplace operator in these coordinates has the form

$$
\Delta=\frac{4}{R^{2}\left(\mu^{2}-\nu^{2}\right)}\left[\frac{\partial}{\partial \mu}\left(\mu^{2}-1\right) \frac{\partial}{\partial \mu}+\frac{\partial}{\partial \nu}\left(1-\nu^{2}\right) \frac{\partial}{\partial \nu}\right]+\frac{4}{R^{2}\left(\mu^{2}-1\right)\left(1-\nu^{2}\right)} \frac{\partial^{2}}{\partial \varphi^{2}} .
$$

After the substitution

$$
\psi(\mu, \nu, \varphi)=\psi_{1}(\mu) \psi_{2}(\nu) \frac{e^{i(m-s) \varphi}}{\sqrt{2 \pi}}
$$

the variables in the Schrödinger equation (1.1) are separated

$$
\begin{gathered}
{\left[\frac{d}{d \mu}\left(\mu^{2}-1\right) \frac{d}{d \mu}+\frac{m_{1}^{2}}{2(\mu+1)}-\frac{m_{2}^{2}}{2(\mu-1)}+R \mu+\frac{E R^{2}}{2}\left(\mu^{2}-1\right)\right] \psi_{1}=\lambda(R) \psi_{1},} \\
{\left[\frac{d}{d \nu}\left(1-\nu^{2}\right) \frac{d}{d \nu}-\frac{m_{1}^{2}}{2(1+\nu)}-\frac{m_{2}^{2}}{2(1-\nu)}-R \nu+\frac{E R^{2}}{2}\left(1-\nu^{2}\right)\right] \psi_{2}=-\lambda(R) \psi_{2},}
\end{gathered}
$$

where $\lambda(R)$ is a separation constant in prolate spheroidal coordinates. By eliminating the the energy $E$ from Eqs.(5.4) and (5.5), we produce the operator

$$
\begin{array}{r}
\hat{\Lambda}=\frac{1}{\mu^{2}-\nu^{2}}\left[\left(1-\nu^{2}\right) \frac{\partial}{\partial \mu}\left(\mu^{2}-1\right) \frac{\partial}{\partial \mu}-\left(\mu^{2}-1\right) \frac{\partial}{\partial \nu}\left(1-\nu^{2}\right) \frac{\partial}{\partial \nu}\right]+ \\
+\frac{2-\mu^{2}-\nu^{2}}{\left(\mu^{2}-1\right)\left(1-\nu^{2}\right)} \frac{\partial^{2}}{\partial \varphi^{2}}+2 s \frac{(\mu+\nu)^{2}-(\mu+1)(1+\nu)}{(\mu+\nu)(\mu-1)(1-\nu)}\left(s+i \frac{\partial}{\partial \varphi}\right)+ \\
+2 c_{1} \frac{(\mu+\nu)^{2}+(\mu-1)(1-\nu)}{(\mu+\nu)(\mu+1)(1+\nu)}+2 c_{2} \frac{(\mu+\nu)^{2}-(\mu+1)(1+\nu)}{(\mu+\nu)(\mu-1)(1-\nu)}+R \frac{\mu \nu+1}{\mu+\nu},
\end{array}
$$

the eigenvalues of which are $\lambda(R)$ and the eigenfunctions of which are $\psi(\mu, \nu, \varphi)$. The significance of the selfadjoint operator $\hat{\Lambda}$ can be found by switching to Cartesian coordinates. Passing to the Cartesian coordinates in (5.7) and taking (2.15) and (2.29) into account, we obtain

$$
\hat{\Lambda}=\hat{M}+R \hat{X} .
$$

Therefore,

$$
\hat{\Lambda} \psi_{n q m}^{(s)}\left(\mu, \nu, \varphi ; R, \delta_{1}, \delta_{2}\right)=\lambda_{q}(R) \psi_{n q m}^{(s)}\left(\mu, \nu, \varphi ; R, \delta_{1}, \delta_{2}\right),
$$

where index $q$ labels the eigenvalues of the operator $\hat{\Lambda}$ and varies in the range $0 \leq q \leq n-m_{+}-1$.

We are now ready to deal with the interbasis expansions

$$
\begin{gathered}
\psi_{n q m}^{(s)}\left(\mu, \nu, \varphi ; R, \delta_{1}, \delta_{2}\right)=\sum_{j=m_{+}}^{n-1} U_{n q m s}^{j}\left(R ; \delta_{1}, \delta_{2}\right) \psi_{n j m}^{(s)}\left(r, \theta, \varphi ; \delta_{1}, \delta_{2}\right) . \\
\psi_{n q m}^{(s)}\left(\mu, \nu, \varphi ; R, \delta_{1}, \delta_{2}\right)=\sum_{n_{1}=0}^{n-m_{+}-1} V_{n q m s}^{n_{1}}\left(R ; \delta_{1}, \delta_{2}\right) \psi_{n_{1} n_{2} m}^{(s)}\left(\xi, \eta, \varphi ; \delta_{1}, \delta_{2}\right) .
\end{gathered}
$$

for the prolate spheroidal basis in terms of the spherical and parabolic bases. (Eq. (5.9) was first considered by Coulson and Joseph 28 in the particular case $s=\delta_{1}=\delta_{2}=0$.)

First, we consider Eq. (5.9). Let the operator $\hat{\Lambda}$ act on both sides of (5.9). Then, by using Eqs. (5.7), (5.8), and (2.12) as well as the orthonormality property of the spherical basis, we find that

$$
\left[\lambda_{q}(R)-\left(j+\frac{\delta_{1}+\delta_{2}}{2}\right)\left(j+\frac{\delta_{1}+\delta_{2}}{2}+1\right)\right] U_{n q m s}^{j}\left(R ; \delta_{1}, \delta_{2}\right)=R \sum_{j^{\prime}=m_{+}}^{n-1} U_{n q m s}^{j^{\prime}}\left(R ; \delta_{1}, \delta_{2}\right)(\hat{X})_{j j^{\prime}},
$$


where

$$
(\hat{X})_{j j^{\prime}}=\int \psi_{n j m}^{(s) *}\left(r, \theta, \varphi ; \delta_{1}, \delta_{2}\right) \hat{X} \psi_{n j m}^{(s)}\left(r, \theta, \varphi ; \delta_{1}, \delta_{2}\right) d V
$$

The calculation of the matrix element $(\hat{X})_{j j^{\prime}}$ can be done by expanding the basis in (5.12) in terms of the parabolic wavefunctions [see Eq. (4.13)] and by making use of the eigenvalue equation for $\hat{X}$ [see Eq. (2.28)]. This leads to

$$
(\hat{X})_{j j^{\prime}}=\frac{2}{2 n+\delta_{1}+\delta_{2}} \sum_{n_{1}=0}^{n-m_{+}-1}\left(2 n_{1}-n+|m-s|+\frac{\delta_{1}+\delta_{2}}{2}+1\right) \tilde{W}_{n j m}^{n_{1}} \tilde{W}_{n j^{\prime} m}^{n_{1}} .
$$

Then, by using Eq. (4.14) together with the recursion relation 25]

$$
\begin{gathered}
C_{a \alpha ; b \beta}^{c \gamma}=-\left[\frac{4 c^{2}(2 c+1)(2 c-1)}{(c+\gamma)(c-\gamma)(b-a+c)(a-b+c)(a+b-c+1)(a+b+c+1)}\right]^{1 / 2} \times \\
\times\left\{\left[\frac{(c-\gamma-1)(c+\gamma-1)(b-a+c-1)(a-b+c-1)(a+b-c+2)(a+b+c)}{4(c-1)^{2}(2 c-3)(2 c-1)}\right]^{1 / 2} \times\right. \\
\left.\times C_{a \alpha ; b \beta}^{c-2, \gamma}-\frac{(\alpha-\beta) c(c-1)-\gamma a(a+1)+\gamma b(b+1)}{2 c(c-1)} C_{a \alpha ; b \beta}^{c-1, \gamma}\right\},
\end{gathered}
$$

and the orthonormality condition

$$
\sum_{\alpha+\beta=\gamma} C_{a \alpha ; b \beta}^{c \gamma} C_{a \alpha ; b \beta}^{c^{\prime} \gamma^{\prime}}=\delta_{c^{\prime} c} \delta_{\gamma^{\prime} \gamma}
$$

we find that $(\hat{X})_{j j^{\prime}}$ is given by

$$
(\hat{X})_{j j^{\prime}}=-\frac{2}{2 n+\delta_{1}+\delta_{2}}\left(A_{n m}^{j+1} \delta_{j^{\prime}, j+1}+A_{n m}^{j} \delta_{j^{\prime}, j-1}\right)+\frac{\left(m_{1}+m_{2}\right)\left(m_{1}-m_{2}\right)}{\left(2 j+\delta_{1}+\delta_{2}\right)\left(2 j+\delta_{1}+\delta_{2}+2\right)} \delta_{j^{\prime}, j},
$$

where

$$
A_{n m}^{j}=\left[\frac{\left(j-m_{+}\right)\left(j+m_{+}+\delta_{1}+\delta_{2}\right)\left(j-m_{-}+\delta_{1}\right)\left(j+m_{-}+\delta_{2}\right)(n-j)\left(n+j+\delta_{1}+\delta_{2}\right)}{\left(j+\frac{\delta_{1}+\delta_{2}}{2}\right)^{2}\left(2 j+\delta_{1}+\delta_{2}-1\right)\left(2 j+\delta_{1}+\delta_{2}+1\right)}\right]^{1 / 2} .
$$

Now by introducing (5.16) into (5.11), we get the following three-term recursion relation for the coefficient $U_{n q m s}^{j}$

$$
\begin{aligned}
& {\left[\lambda_{q}(R)-\left(j+\frac{\delta_{1}+\delta_{2}}{2}\right)\left(j+\frac{\delta_{1}+\delta_{2}}{2}+1\right)-\frac{R\left(m_{1}+m_{2}\right)\left(m_{1}-m_{2}\right)}{\left(2 j+\delta_{1}+\delta_{2}\right)\left(2 j+\delta_{1}+\delta_{2}+2\right)}\right] U_{n q m s}^{j}\left(R ; \delta_{1}, \delta_{2}\right)+} \\
& +\frac{2 R}{2 n+\delta_{1}+\delta_{2}}\left[A_{n m}^{j+1} U_{n q m s}^{j+1}\left(R ; \delta_{1}, \delta_{2}\right)+A_{n m}^{j} U_{n q m s}^{j-1}\left(R ; \delta_{1}, \delta_{2}\right)\right]=0 .
\end{aligned}
$$

The recursion relation (5.18) provides us with a system $n-m_{+}$linear homogeneous equations which can be solved by taking into account the normalization condition

$$
\sum_{j-m_{+}}^{n-1}\left|U_{n q m s}^{j}\left(R ; \delta_{1}, \delta_{2}\right)\right|^{2}=1
$$

Th eigenvalues $\lambda_{q}(R)$ of the operator $\hat{\Lambda}$ then follow from the vanishing of the determinant for the latter system. 
Second, let us concentrate on the expansion (5.10) of the prolate spheroidal basis in terms of the parabolic basis. By employing technique similar to the one used for deriving Eq. (5.11), we get

$$
\left[\lambda_{q}(R)-\frac{2 R}{2 n+\delta_{1}+\delta_{2}}\left(n_{1}-n_{2}+\frac{m_{1}-m_{2}}{2}\right)\right] V_{n q m s}^{n_{1}}\left(R ; \delta_{1}, \delta_{2}\right)=\sum_{n_{1}^{\prime}=0}^{n-m_{+}-1} V_{n q m s}^{n_{1}^{\prime}}\left(R ; \delta_{1}, \delta_{2}\right)(\hat{M})_{n_{1} n_{1}^{\prime}},(5
$$

where

$$
(\hat{M})_{n_{1} n_{1}^{\prime}}=\int \psi_{n_{1} n_{2} m}^{(s) *}\left(\xi, \eta, \varphi ; \delta_{1}, \delta_{2}\right) \hat{M} \psi_{n_{1}^{\prime} n_{2}^{\prime} m}^{(s)}\left(\xi, \eta, \varphi ; \delta_{1}, \delta_{2}\right) d V
$$

The matrix elements $(\hat{M})_{n_{1} n_{1}^{\prime}}$ can be calculated in the same way as $(\hat{X})_{j j^{\prime}}$ except that now we must use the relation 29$]$

$$
\begin{array}{r}
{[c(c+1)-a(a+1)-b(b+1)-2 \alpha \beta] C_{a, \alpha ; b, \beta}^{c, \gamma}=} \\
=\sqrt{(a+\alpha)(a-\alpha+1)(b-\beta)(b+\beta+1)} C_{a, \alpha-1 ; b, \beta+1}^{c, \gamma}+ \\
+\sqrt{(a-\alpha)(a+\alpha+1)(b+\beta)(b-\beta+1)} C_{a, \alpha+1 ; b, \beta-1}^{c, \gamma},
\end{array}
$$

and the orthonormality condition

$$
\sum_{c=|\gamma|}^{a+b} C_{a \alpha ; b \beta}^{c \gamma} C_{a \alpha^{\prime} ; b \beta^{\prime}}^{c \gamma}=\delta_{\alpha \alpha^{\prime}} \delta_{\beta \beta^{\prime}}
$$

permit deriving the formula for the matrix element $(\hat{M})_{n_{1} n_{1}^{\prime}}$

$$
\begin{array}{r}
(\hat{M})_{n_{1} n_{1}^{\prime}}=\left[\left(n_{1}+1\right)\left(n_{2}+m_{-}\right)+\left(n-n_{1}+\delta_{2}\right)\left(n_{1}+|m-s|+\delta_{2}\right)+\frac{1}{4}\left(\delta_{1}-\delta_{2}\right)\left(\delta_{1}-\delta_{2}-2\right)+\right. \\
\left.+m_{-}\left(m_{+}+\delta_{2}\right)\right] \delta_{n_{1}^{\prime} n_{1}}-\sqrt{n_{2}\left(n_{1}+1\right)\left(n_{1}+|m-s|+\delta_{1}+1\right)\left(n_{2}+|m-s|+\delta_{2}\right)} \delta_{n_{1}^{\prime}, n_{1}+1} \\
-\sqrt{n_{1}\left(n_{2}+1\right)\left(n_{1}+|m-s|+\delta_{1}+1\right)\left(n_{2}+|m-s|+\delta_{2}+1\right)} \delta_{n_{1}^{\prime}, n_{1}-1}
\end{array}
$$

Finally, the introduction of (5.25) into (5.20) leads to the three-term recursion relation

$$
\begin{aligned}
& {\left[\left(n_{1}+1\right)\left(n_{2}+m_{-}\right)+\left(n-n_{1}+\delta_{2}\right)\left(n_{1}+|m-s|+\delta_{2}\right)+\frac{1}{4}\left(\delta_{1}-\delta_{2}\right)\left(\delta_{1}-\delta_{2}-2\right)+\right.} \\
& \left.+m_{-}\left(m_{+}+\delta_{2}\right)+\frac{2 R}{2 n+\delta_{1}+\delta_{2}}\left(n_{1}-n_{2}+\frac{m_{1}-m_{2}}{2}\right)-\lambda_{q}(R)\right] V_{n q m s}^{n_{1}}\left(R ; \delta_{1}, \delta_{2}\right)= \\
& \sqrt{n_{2}\left(n_{1}+1\right)\left(n_{1}+|m-s|+\delta_{1}+1\right)\left(n_{2}+|m-s|+\delta_{2}\right)} V_{n q m s}^{n_{1}+1}\left(R ; \delta_{1}, \delta_{2}\right)+ \\
& +\sqrt{n_{1}\left(n_{2}+1\right)\left(n_{1}+|m-s|+\delta_{1}+1\right)\left(n_{2}+|m-s|+\delta_{2}+1\right)} V_{n q m s}^{n_{1}-1}\left(R ; \delta_{1}, \delta_{2}\right)
\end{aligned}
$$

for the expansion coefficients $V_{n q m s}^{n_{1}}\left(R ; \delta_{1}, \delta_{2}\right)$. This relation can be iterated by taking account of the normalization condition

$$
\sum_{n_{1}=0}^{n-m_{+}-1}\left|V_{n q m s}^{n_{1}}\left(R ; \delta_{1}, \delta_{2}\right)\right|^{2}=1
$$

Here again, the eigenvalues $\lambda_{q}(R)$ may be obtained by solving a system of $n-m_{+}$linear homogeneous equations.

It should be mentioned, that the formulae (4.12) and (4.14) and three-term recursion relations (5.18) and (5.18) generalize the analogical results for the following systems: 
- Hydrogen atom [30, 31, 32, 27, 28, 33, when $s=\delta_{1}=\delta_{2}=0$.

- Generalized Kepler-Coulomb system [17, when $s=0, \delta_{1} \neq \delta_{2} \neq 0$.

- Hartmann system [34, when $s=0, \delta_{1}=\delta_{2} \neq 0$.

- Charge-dyon system $\left[8\right.$, when $s \neq 0, \delta_{1}=\delta_{2}=0$.

Finally, it should be noted that the following four limits

$$
\begin{aligned}
& \lim _{R \rightarrow 0} U_{j n q m s}^{j}\left(R ; \delta_{1}, \delta_{2}\right)=\delta_{j q}, \quad \lim _{R \rightarrow \infty} U_{n q m s}^{j}\left(R ; \delta_{1}, \delta_{2}\right)=W_{n_{1} n_{2} m s}^{j}\left(\delta_{1}, \delta_{2}\right), \\
& \lim _{R \rightarrow \infty} V_{n q m s}^{n_{1}}\left(R ; \delta_{1}, \delta_{2}\right)=\delta_{n_{1} q}, \quad \lim _{R \rightarrow 0} V_{n q m s}^{n_{1}}\left(R ; \delta_{1}, \delta_{2}\right)=\tilde{W}_{n j m s}^{n_{1}}\left(\delta_{1}, \delta_{2}\right)
\end{aligned}
$$

furnish a useful means for checking the calculations presented in Sections 4 and 5.

Acknowledgements. I would like to thank Dr. Armen Nersessian for useful discussions. The work is carried out with the support of ANSEF No: PS81 grant.

\section{References}

[1] L.G. Mardoyan. The generalized MIC-Kepler system. quant-ph/ 0306168; J. Math. Phys., 44, No. 12 (2003) (in press).

[2] D. Zwanziger. Phys. Rev., 176, 1480 (1968).

[3] H. McIntosh and A. Cisneros. J. Math. Phys., 11, 896 (1970).

[4] T. Iwai and Y. Uwano. J. Phys. A. 21, 4083 (1988). A. Nersessian and V. Ter-Antonyan. Mod. Phys. Lett. A. 9, 2431 (1994); Mod. Phys. Lett. A., 10, 2633 (1995).

[5] A. Nersessian, V. Ter-Antonyan, M.M. Tsulaia. Mod. Phys. Lett. A. 11, 1605 (1996); A.P. Nersessian, V.M. Ter-Antonyan. Physics of Atomic Nuclei, 61, 1756 (1998).

[6] T.Iwai. J. Geom. Phys., 7, 507 (1990). L.G. Mardoyan, A.N. Sissakian and V.M. Ter-Antonyan. Physics of Atomic Nuclei, 61, 1746 (1998);

[7] C.N. Yang. J. Math. Phys., 19, 320 (1978).

[8] L.G. Mardoyan, A.N. Sissakian and V.M. Ter-Antonyan. Int. J. Mod. Phys. A. 12, 237 (1997).

[9] L.G. Mardoyan, A.N. Sissakian and V.M. Ter-Antonyan. Theor. and Math. Phys., 123, 451 (2000); L.G. Mardoyan. Physics of Atomic Nuclei, 65, 1063 (2002).

[10] V.V. Gritsev, Yu.A. Kurochkin and V.S. Otchik, J. Phys. A. 33, 4903 (2000).

[11] A. Nersessian and G. Pogosyan. Phys. Rev. A. 63, 020103(R) (2001).

[12] I. Mladenov and V. Tsanov. J. Phys. A 20, 5865 (1987).

[13] T. Iwai and Y. Uwano. J. Math. Phys. 27, 1523 (1986).

[14] A. Inomata, G. Junker, and R. Wilson. Found. Phys. 23, 1073 (1993).

[15] S. Bellucci and A. Nersessian. Phys. Rev. D, 67, 065013 (2003).

[16] L.G. Mardoyan, L.S. Petrosyan, and H.A. Sarkisyan. Phys. Rev. A, 68, 014103 (2003).

[17] M. Kibler, L.G. Mardoyan and G.S. Pogosyan. Int. J. Quan. Chem., 52, 1301 (1994). 
[18] H. Hartmann. Theor. Chim. Acta 24, 201 (1972); H. Hartmann, R. Schuch, and J. Radke. Theor. Chim. Acta 42, 1 (1976); H. Hartmann and R. Schuch. Int. J. Quant. Chem. 18, 125(1980).

[19] J. Fris, V. Mandrosov, Ya.A. Smorodinsky, M. Uhlir, and P. Winternitz. Phys. Lett. 16, 354, (1965); P. Winternitz, Ya.A. Smorodinsky, M. Uhlir, and J. Fris. Sov. J. Nucl. Phys. 4, 444, (1967); A.A. Makarov, J.A. Smorodinsky, Kh. Valiev, and P. Winternitz. Nuovo Cimento A 52, 1061, (1967).

[20] N.W. Evans. Phys. Lett. A 147, 483, (1990); Phys. Rev. a 41, 5666, (1990); J. Math. Phys. 31, 600, (1990).

[21] I.V. Komarov, L.I. Ponomarev, and S.Yu. Slavyanov. Spheroidal and Coulomb Spheroidal Functions (Nauka, Moscow, 1976).

[22] L.D. Landau, E.M. Lifshitz. Quantum Mechanics (Pergamon Press, Oxford, 1977).

[23] A. Erdelyi, W. Magnus, F. Oberhettinger, and F. Tricomi. Higher Transcendental Functions (McGrawHill, New-York, 1953) Vol. I.

[24] L.G. Mardoyan, G.S. Pogosyan, and V.M. Ter-Antonyan. Sov. Contemp. Phys., 19, 3 (1984).

[25] D.A. Varshalovich, A.N. Moskalev, and V.K. Khersonskii. Quantum Theory of Angular Momentum. World Scientific, Singapore, (1988).

[26] W.N. Bailey. Generalized Hypergeometric Series. Cambridge Tracts No. 32, Cambridge, (1935).

[27] L.G. Mardoyan, G.S. Pogosyan, A.N. Sissakian, and V.M. Ter-Antonyan. J. Phys. A16, 711 (1983).

[28] C.A. Coulson and A. Joseph. Proc. Phys. Soc., 90, 887 (1967).

[29] M. Kibler, G. Grenet. J. Math. Phys., 21, 422 (1980).

[30] D. Park. Z. Phys., 159, 155 (1960).

[31] C.B. Tarter. J. Math. Phys., 11, 3192 (1970).

[32] M.G. Arutyunyan, G.S Pogosyan, and V.M. Ter-Antonyan. Sov. Contemp. Phys., 13, 235 (1978).

[33] L.G. Mardoyan, G.S. Pogosyan, A.N. Sissakian, and V.M. Ter-Antonyan. Theor. Math. Phys., 64, 171 (1985).

[34] I.V. Lutsenko, G.S. Pogosyan, A.N. Sissakian, and V.M. Ter-Antonyan. Theor. Math. Phys., 83, 419 (1990). 